\title{
Changes in self-reported physical activity and health-related quality of life following 3-month astaxanthin supplementation in patients with heart failure: results from a pilot study
}

\author{
Sayaki Ishiwata ${ }^{1,2 \#}$, Takao Kato ${ }^{1 \#}$, Takatoshi Kasai ${ }^{1,2,3} \wedge$, Akihiro Sato ${ }^{1,2}$, Shoichiro Yatsu ${ }^{1}$, Hiroki Matsumoto ${ }^{1}$, \\ Jun Shitara ${ }^{1}$, Azusa Murata ${ }^{1}$, Megumi Shimizu ${ }^{1}$, Shoko Suda ${ }^{1}$, Yuya Matsue ${ }^{1,2}$, Ryo Naito ${ }^{1,2}$, Masaru Hiki ${ }^{1}$, \\ Hiroyuki Daida ${ }^{1}$
}

${ }^{1}$ Department of Cardiovascular Medicine, Juntendo University School of Medicine, Tokyo, Japan; ${ }^{2}$ Cardiovascular Respiratory Sleep Medicine, Juntendo University Graduate School of Medicine, Tokyo, Japan; ${ }^{3}$ Sleep and Sleep Disordered Breathing Center, Juntendo University Hospital, Tokyo, Japan

Contributions: (I) Conception and design: T Kato, T Kasai; (II) Administrative support: H Daida; (III) Provision of study material or patients: All authors; (IV) Collection and assembly of data: All authors; (V) Data analysis and interpretation: S Ishiwata, T Kato, T Kasai; (VI) Manuscript writing: All authors; (VII) Final approval of manuscript: All authors.

\#These authors contributed equally to this work.

Correspondence to: Takatoshi Kasai, MD, PhD. Department of Cardiovascular Medicine, Juntendo University Graduate School of Medicine; Cardiovascular Respiratory Sleep Medicine, Juntendo University Graduate School of Medicine; Sleep and Sleep Disordered Breathing Center, Juntendo University Hospital, 2-1-1 Hongo, Bunkyo-ku, Tokyo 113-8421, Japan. Email: kasai-t@mx6.nisiq.net.

Background Astaxanthin has a strong antioxidant effect. We recently demonstrated that following 3-month
astaxanthin supplementation, cardiac contractility and exercise tolerance improved, possibly through the
suppression of oxidative stress in a small pilot study involving patients with heart failure with left ventricular
systolic dysfunction. This is a sub-study of our pilot study to investigate whether improvements of self-
reported physical activity and health-related quality of life were observed following 3-month astaxanthin
supplementation.

Methods: We investigated the changes in physical activity by the Specific Activity Scale score and healthrelated quality of life by physical and mental component summary scores in Short Form-8 at baseline and after 3-month astaxanthin supplementation.

Results: Data from 17 patients with heart failure were assessed. Following 3-month astaxanthin supplementation, the Specific Activity Scale score increased from the median of 4.5 (interquartile range, $2.0)$ to 6.5 (interquartile range, 1.1$)$ metabolic equivalent $(\mathrm{P}=0.001)$, and the physical and mental component summary scores increased from $46.1 \pm 9.2$ to $50.8 \pm 6.8(\mathrm{P}=0.015)$ and from $48.9 \pm 9.1$ to $53.8 \pm 4.8(\mathrm{P}=0.022)$, respectively. There was a linear relationship of the baseline heart rate, or mental component summary score with the percent change in the Specific Activity Scale score ( $r=0.523, \mathrm{P}=0.031$ and $\mathrm{r}=-0.505, \mathrm{P}=0.039$, respectively). In addition, there was a direct relationship of ischemic etiology with the percent change in the physical component summary score ( $\mathrm{r}=0.483, \mathrm{P}=0.049$, respectively). Finally, there was a linear relationship between the percent change in the Specific Activity Scale score and that in the mental component summary score ( $\mathrm{r}=0.595, \mathrm{P}=0.012)$.

Conclusions: Following 3-month astaxanthin supplementation, improvements of the self-reported physical activity level and health-related quality of life in both mental and physical components were observed. In patients with heart failure, those with higher baseline heart rate, ischemic etiology, and poorer baseline health-related quality of life have potentials to have greater improvement of physical activity and/or

^ ORCID: 0000-0001-5747-7668. 
health-related quality of life.

Keywords: Antioxidants; health-related quality of life; oxidative stress; physical activity; Specific Activity Scale

Submitted Jul 08, 2020. Accepted for publication Sep 10, 2020.

doi: 10.21037/apm-20-1378

View this article at: http://dx.doi.org/10.21037/apm-20-1378

\section{Introduction}

Reportedly, astaxanthin, which is generally containing in natural sea products including crab, shrimp, salmon, and salmon roe (1), has a strong antioxidant property with a potency $>100$-fold greater compared with that of $\alpha$-tocopherol (2) and indeed inhibits oxidative damage and its related systemic inflammatory responses, both of which are the established cardiovascular risk factors and are regarded as potential target for intervention. Previous studies showed that supplementation of astaxanthin decreased the plasma oxidative stress maker levels and serum of pro-inflammatory cytokines levels such as C-reactive protein (CRP) and tumor necrosis factor (TNF)- $\alpha$ in healthy women or overweight/obese subjects $(3,4)$. Therefore, astaxanthin is thought to have protective effects of the cardiovascular system. However, there are limited data regarding the improvements of clinical cardiovascular parameters by astaxanthin supplementation. Other data have suggested that astaxanthin has beneficial effects on exercise tolerance due to its antioxidant properties (5-7). These findings suggest that astaxanthin supplement is beneficial for exercise tolerance particularly in subjects with increased oxidative stress.

In this regard, in patients with heart failure (HF) who generally suffer from increased oxidative stress (8), astaxanthin supplementation may benefit the improvement of exercise tolerance. We recently showed that 3-month astaxanthin supplementation may improve cardiac contractility and exercise tolerance, possibly through the suppression of oxidative stress in a small pilot study involving patients with HF with left ventricular (LV) systolic dysfunction (9). In terms of exercise tolerance, most of the exercise tests are aiming to evaluate exercise performance at maximal workloads, but daily physical activities do not require energy expenditure in the maximal range particularly in patients with HF. Therefore, the improvement of exercise tolerance observed in our previous study should be confirmed by other measures that can allow the expression of the extent of submaximal physical activities.

In patients with HF, health-related quality of life (HRQoL) is impaired due to various reasons, including limited physical activity (10). If physical activity can be improved by astaxanthin supplementation, HRQoL may also be improved. Therefore, we conducted a sub-analysis of our previous study to assess the potential effects of 3-month astaxanthin supplementation on self-reported physical activity, which can allow the expression of the extent of submaximal physical activities and HRQoL in patients with HF with systolic dysfunction. Our specific hypothesis is that following 3 -month astaxanthin supplementation improvements of self-reported physical activity and HRQoL were observed in patients with HF. We present the following article in accordance with the TREND reporting checklist (available at http://dx.doi.org/10.21037/apm-201378).

\section{Methods}

\section{Subjects}

We enrolled patients with systolic HF at Juntendo University Hospital (Tokyo, Japan) if they met the following criteria: (I) men and women aged $\geq 20$ years, (II) HF due to ischemic or nonischemic cardiomyopathy, (III) left ventricular ejection fraction (LVEF) $<50 \%$ on echocardiography, (IV) New York Heart Association functional class $\geq \mathrm{II}$, and (V) stable condition as evidenced by the lack of symptoms related to the acute exacerbation of HF. The exclusion criteria included: patients who could not undergo the 6-min walk test; those who could not answer a questionnaire by themselves; those who underwent cardiac surgery during 4 weeks prior to the enrollment; those with acute coronary syndrome, organic valvular heart diseases, chronic inflammatory diseases, chronic lung diseases, or malignancy; those under dialysis; and current smokers. This is a sub-study of the prospective observational pilot study (UMIN-ID: 000014088), which was approved by the Juntendo University Hospital Institutional Review 
Board (\#14-019), and the study complied with the ethical principles of the Declaration of Helsinki (as revised in 2013). Written informed consent was obtained from all participants.

\section{Anthropometric, bemodynamic parameters and blood samplings}

Height and weight were measured in the morning of the day before starting the astaxanthin supplementation, and body mass index was computed. Blood pressure (BP) and heart rate (HR) were also measured at the same time. Blood samples were obtained in the morning after an overnight fast. Serum oxidative stress was assessed by the Diacron reactive oxygen metabolites ( $\mathrm{dROM}$ ) test, which measures the total oxidant capacity of samples relative to the chromogenic substrate N,N-diethyl-p-phenylenediamine. Total antioxidant capacity was assessed by the biological antioxidant potential (BAP) test, which measures the capacity of a sample to reduce ferric ions to ferrous ions with a thiocyanate derivative as a chromogen. A free radical analyzer system (Free Carrio Duo, Wismerll Company, Ltd., Tokyo, Japan) was used for dROM and BAP tests. The urinary oxidative stress marker measurement system, ICR001 (Immunochromatography method, Techno Medica Co.) was used for urinary 8-hydroxy-2'-deoxyguanosine (8-OHdG) concentrations with a measurement of the urinary creatinine level. and the urinary $8-\mathrm{OHdG}(\mathrm{ng} / \mathrm{mL}) /$ creatinine $(\mathrm{mg} / \mathrm{mL})$ ratio was reported. As inflammatory markers, in addition to the CRP, the serum levels of TNF- $\alpha$ were assayed using a high sensitive Enzyme-Linked ImmunoSorbent Assay kit (R\&D Systems, Minneapolis, USA). The estimated glomerular filtration rate was computed based on the serum level of creatinine (11). The plasma concentration of astaxanthin was measured via high-performance liquid chromatography whose details were previously described (12). For the cardiac function, the LVEF was assessed by the modified Simpson method. Echocardiographic studies were done and assessed by experienced sonographer and cardiologists who were blinded to the clinical data. Plasma B-type natriuretic peptide level was assessed.

\section{Questionnaire-based physical activity and HRQoL}

As a self-report-based physical activity scale, we used the Specific Activity Scale (SAS) with all patients being asked 21 questions, as a measure of self-perceived exercise tolerance (13). SAS scores are expressed as metabolic equivalent (Mets). Most of the exercise tests were aiming to assess exercise performance around maximal workloads, but daily activities did not require energy expenditure in the maximal range. In this regard, the SAS allowed the expression of the extent of submaximal physical activities. The HRQoL was evaluated by the Short Form (SF)- $8^{\mathrm{TM}}$ consisting of 8 questionnaires. The Japanese version of SF-8 was validated $(14,15)$. There are eight subscales, such as physical functioning, role limitations due to physical problems (role-physical), bodily pain, general health perception, vitality, social functioning, role limitations due to emotional problems (role-emotional), and mental health. Two aggregate scores, the physical component summary (PCS) and mental component summary (MCS) scores, were computed from the eight subscales. These scores were computed by weighing each subscale (15). Scoring was based on the Japanese standards; the possible scores range from 0 to 100 , with higher scores representing a better HRQoL (15).

\section{Astaxanthin supplement and study protocol}

Patients took astaxanthin supplement containing $12 \mathrm{mg}$ of astaxanthin with $40 \mathrm{mg}$ of tocotrienol (vitamin E) and $30 \mathrm{mg}$ of L-ascorbic acid 2-glucoside (vitamin C) orally (AstaReal ACT, AstaReal Co., Ltd., Tokyo, Japan) for 3 months. No changes in other medications were allowed in the study periods. The measurements of body weight, systolic and diastolic BP, and HR were obtained at baseline and followup clinic visit. Blood sampling, LVEF assessment, and questionnaires (SAS and SF-8) were repeated at 3 months after starting astaxanthin supplementation.

\section{Statistical analysis}

Because no data regarding the effects of astaxanthin supplement in patients with HF were available, we did not compute the specific sample size and conduct the present study as a pilot study. Nevertheless, we planned to perform analysis at the time when $\geq 15$ patients completed the 3 -month follow-up period. Values are expressed as mean \pm SD unless indicated otherwise. The differences between the baseline and follow-up measurements were compared using the paired t-test for normally distributed data and the Wilcoxon signed-rank test for non-normally distributed data. Changes in parameters from baseline to 3 months (i.e., $\Delta)$ were computed as $\Delta=[($ values at 
Table 1 Baseline characteristics $(\mathrm{N}=17)$

\begin{tabular}{lc}
\hline Characteristics & Data \\
\hline Age, years & $67.8 \pm 14.5$ \\
BMI, $\mathrm{kg} / \mathrm{m}^{2}$ & $23.2 \pm 3.7$ \\
Women, $\mathrm{n}(\%)$ & $3(17.6)$ \\
NYHA class II, $\mathrm{n}(\%)$ & \\
II & $16(94.1)$ \\
III & $1(5.9)$ \\
LVEF, \% & $33.8 \pm 8.4$ \\
Ischemic heart disease, $\mathrm{n}(\%)$ & $8(47.1)$ \\
Diabetes mellitus, $\mathrm{n}(\%)$ & $5(29.4)$ \\
eGFR $\leq 60$ mL/min/1.73 ${ }^{2}, \mathrm{n}(\%)$ & $11(64.7)$ \\
Use of ACE-Is/ARBs, $\mathrm{n}(\%)$ & $16(94.1)$ \\
Use of beta blockers, $\mathrm{n}(\%)$ & $17(100.0)$ \\
Use of MR antagonists, $\mathrm{n}(\%)$ & $12(70.6)$ \\
Use of diuretics, $\mathrm{n}(\%)$ & $13(76.5)$
\end{tabular}

BMI, body mass index; ACE-ls, angiotensin-converting enzyme inhibitors; ARBs, angiotensin II receptor blockers; BMI, body mass index; eGFR, estimated glomerular filtration rate; MR, mineral corticoid receptor; LVEF, left ventricular ejection fraction; NYHA, New York Heart Association.

3 months)-(values at baseline) $]$ and expressed as $\% \Delta[\Delta /$ (values at baseline) $\times 100$ ], except for plasma astaxanthin concentration because astaxanthin was not detected at baseline in most participants. Relationships between the baseline parameters and $\% \Delta$ in the SAS, PCS, and MCS scores and relationships across $\% \Delta$ in parameters were assessed using the Pearson and Spearman correlation coefficients for normally and non-normally distributed data, respectively. A P value less than 0.05 indicated statistical significance. Analyses were performed by SPSS 23.0 (IBM Corp., Armonk, NY, USA).

\section{Results}

\section{Characteristics of all patients}

Nineteen eligible patients were enrolled, all of whom had been diagnosed as HF and treated for at least 6 months at enrollment. However, 2 patients were excluded because of lost to follow-up. Thus, data of 17 patients (14 males, 3 females) who completed oxidative stress and inflammatory marker and LVEF assessments and questionnaires were analyzed. All other medications remained unchanged during the study period. Although 1 patient had worsening of spinal stenosis at follow-up, which was determined not to be related to astaxanthin supplementation, no other complaints or adverse events were observed. The baseline characteristics of these 17 patients are shown in Table 1.

\section{Changes in anthropometric, hemodynamic parameters, blood samples, physical activity, and HRQoL}

From the baseline to the follow-up, the plasma astaxanthin concentration increased significantly from $0(0)$ to 136.0 (135.4) $\mathrm{ng} / \mathrm{mL}(\mathrm{P}<0.001)$. Following 3 -month astaxanthin supplementation, LVEF increased, and dROM decreased significantly (Table 2). However, there were no changes in other parameters (Table 2). The SAS score significantly increased following 3-month astaxanthin supplementation (Figure 1). In addition, both PCS and MCS scores in SF-8 significantly increased following 3 -month astaxanthin supplementation (Figures 2 and 3).

\section{Relationship between baseline parameters and $\% \Delta$ in the SAS and HRQoL scores}

A significant relationship was found between the baseline $\mathrm{HR}$ and $\% \Delta$ in the SAS score $(\mathrm{r}=0.523, \mathrm{P}=0.031)$ (i.e., the greater the baseline $\mathrm{HR}$, the greater the increase in the SAS score). In addition, there was a significant relationship between the baseline MCS score and $\% \Delta$ in the SAS score $(r=-0.505, P=0.039)$ (i.e., the lower the baseline MCS score, the greater the increase in the SAS score). There was a significant relationship between the ischemic etiology and $\% \Delta$ in the PCS score $(r=0.483, P=0.049)$ (i.e., patients with ischemic etiology showed significantly greater increase in the PCS score than those with nonischemic etiology).

\section{Relationships between $\% \Delta$ in parameters and $\% \Delta$ in the SAS and HRQoL scores}

There was a significant direct relationship between $\% \Delta$ in the SAS score and $\% \Delta$ in the MCS score $(r=0.595$, $\mathrm{P}=0.012)$. However, no relationships were found across $\% \Delta$ in other parameters.

\section{Conclusions}

The specific findings of this sub-study also provide some novel insights into the associations of astaxanthin 
Table 2 Changes in body weight, hemodynamic parameters, and blood samples ( $\mathrm{N}=17)$

\begin{tabular}{|c|c|c|c|}
\hline & Baseline & 3 months & $\mathrm{P}$ \\
\hline Systolic blood pressure, $\mathrm{mmHg}$ & $111.2 \pm 18.4$ & $109.5 \pm 16.9$ & 0.647 \\
\hline Diastolic blood pressure, $\mathrm{mmHg}$ & $59.4 \pm 10.1$ & $62.2 \pm 10.2$ & 0.297 \\
\hline Heart rate, beat/min & $70.8 \pm 9.2$ & $67.8 \pm 11.5$ & 0.195 \\
\hline Serum TNF- $\alpha, \mathrm{pg} / \mathrm{mL}$ & $1.7 \pm 0.6$ & $1.7 \pm 0.6$ & 0.844 \\
\hline Serum CRP, mg/dL & $0.1[0.2]$ & $0.1[0.1]$ & 0.526 \\
\hline Serum dROM, U.CARR & $390.4 \pm 82.3$ & $348.7 \pm 55.8$ & 0.018 \\
\hline Plasma BAP, $\mu \mathrm{mol} / \mathrm{L}$ & $2023.0[219.0]$ & 2014.0 [201.3] & 0.586 \\
\hline
\end{tabular}

TNF- $\alpha$, tumor necrosis factor $\alpha$; CRP, C-reactive protein; dROM, Diacron reactive oxygen metabolites; BAP, biological antioxidant potential; 8-OHdG, 8-hydroxy-20-deoxyguanosine; BNP, B-type natriuretic peptide.

supplement with the self-reported physical activity and HRQoL in patients with HF. First, improvements of the self-reported physical activity, SAS score, and HRQoL assessed by SF-8 summary scores were observe following 3 -month astaxanthin supplementation. Second, patients with increased baseline HR or low baseline MCS score were likely to have an improvement of the SAS score. Third, patients with ischemic etiology were likely to have an improvement of the PCS score. Finally, the improvement of the SAS score was correlated directly with the improvement of the MCS score. Taken together, in patients with HF with $\mathrm{LV}$ systolic dysfunction, following 3-month astaxanthin supplementation, improvements of the self-reported physical activity and HRQoL scores were observed and such improvements in the physical activity and HRQoL could be more prominent in patients with rapid HR, ischemic etiology, or HRQoL.

In previous studies in animal models and human subjects, astaxanthin supplementation was shown to have direct and indirect effects through the suppression of oxidative stress on the myocardium and skeletal muscles and consequently may have potential to improve exercise performance in HF patients. Indeed, in our main study, we have shown that the 6-min walk distance increased significantly in patients with HF. In this sub-study, we found that the SAS score, which can allow the expression of the extent of submaximal physical activities, also increased significantly following 3-month astaxanthin supplementation. It is of great interest that patients with greater baseline HR were likely to have more improvement of the SAS score. This is supported by the facts that increased resting HR was associated with increased levels of several surrogate markers and poor clinical outcomes in patients with HF (16) and the reduction of resting HR in patients with HF by beta blockers or ivabradine improved exercise tolerance $(17,18)$. In addition, it should be noted that lower baseline MCS scores could also be a predictor for greater improvement of the SAS score in patients.

Most studies regarding the effects of antioxidants failed to show the improvement of HRQoL in patients with HF except for a few studies (19-22). In this sub-study, we found improvements in both PCS and MCS scores following 3 -month administration of antioxidants (i.e., astaxanthin). One possible explanation for such discrepancy between previous studies and ours may include differences in the type of antioxidants. As mentioned earlier, astaxanthin, one of the carotenoids, has a strong antioxidant effect (2), but unlike other carotenoids which simultaneously have antiand pro- oxidative properties (23), astaxanthin shows solely antioxidative effects (23). Thus, the effects of astaxanthin may be different from other antioxidants and supposed to be greater than the previously investigated antioxidants. The improvements of PCS were more prominent in patients with $\mathrm{HF}$ with ischemic etiology in this sub-study. Based on the results of our sub-study, patients with HF with ischemic etiology may be considered as a potential candidate of 


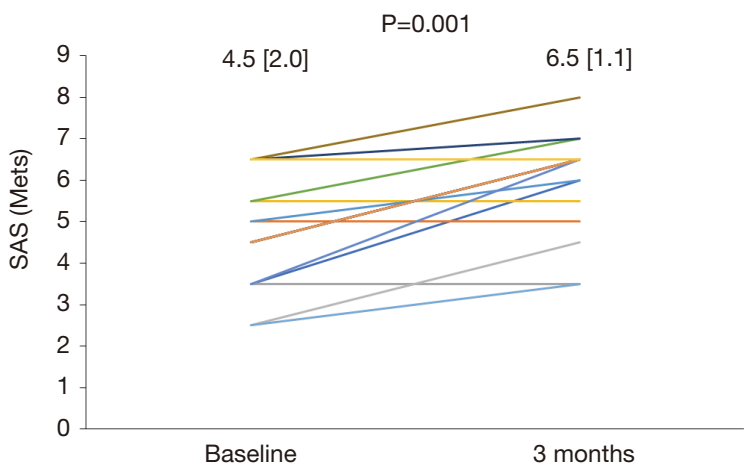

Figure 1 Individual changes in the SAS score. Significant increase in the SAS score was observed following 3-month astaxanthin supplementation. SAS, Specific Activity Scale; Mets, metabolic equivalent.

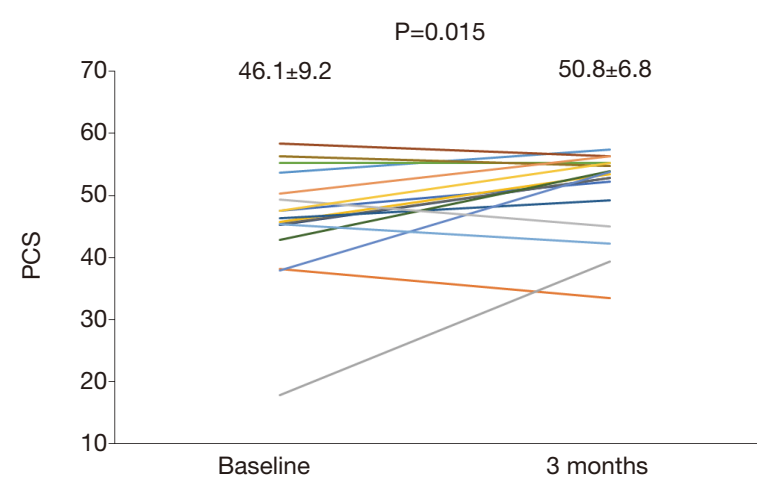

Figure 2 Individual changes in the PCS score. Significant increase in the PCS score was observed following 3-month astaxanthin supplementation. PCS, physical component summary.

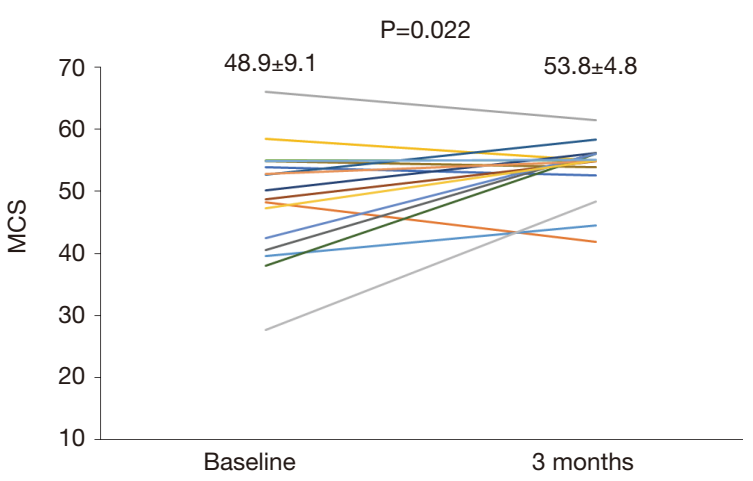

Figure 3 Individual changes in the MCS score. Significant increase in the MCS score was observed following 3-month astaxanthin supplementation. MCS, mental component summary. astaxanthin supplementation to see an improvement of the physical activity and HRQoL in greater extent.

In patients with $\mathrm{HF}$, one reason for reduced HRQoL includes limited physical activity (10). Thus, the improvement of physical activity may positively affect the HRQoL score in general. In this sub-study, indeed, there was a significant direct correlation between the improvement of the SAS score and that of the MCS score. These findings suggest that in patients with HF, following 3-month astaxanthin supplementation, the mental HRQoL scores may increase in association with the improvement of physical activity on submaximal and daily levels. Whether direct effects of astaxanthin supplementation on HRQoL remains to be elucidated.

Our study has several limitations. First, since this was an observational uncontrolled study, causal relationship between astaxanthin supplementation and changes in the self-reported physical activity and HRQoL was not proven. In particular, it should be noted that because no blinding was performed, assessments based on subjective tools could be biased by the placebo effect. Second, in the present study we used the astaxanthin supplement contained $40 \mathrm{mg}$ of tocotrienol and $30 \mathrm{mg}$ of L-ascorbic acid 2-glucoside in addition to $12 \mathrm{mg}$ of astaxanthin. Because of their antioxidant properties, tocotrienol and L-ascorbic acid 2-glucoside may too have the potential to decrease oxidative stress and improve physical activity and HRQoL. However, because their effects on exercise tolerance and HRQoL remain controversial $(19,20)$ and astaxanthin has a stronger effect as antioxidant $(2,24)$, we believe that astaxanthin played the major role in the suppression of oxidative stress and improvement of physical activity and HRQoL. Although there are some forms of astaxanthin supplementation (25), ultimately, these effects observed in the present study could be the result of this astaxanthin supplement containing tocotrienol, L-ascorbic acid 2-glucoside, and astaxanthin. Third, there were only 17 patients. Thus, the number of subjects was small although the present study was a pilot study. Because there are no study investigating the effects of astaxanthin on physical activity and HRQoL in HF patients, a proofof-concept study like this study are needed to generate further hypothesis. In addition, our finding that patients with $\mathrm{HF}$ with higher baseline HR, ischemic etiology, and lower baseline MCS scores were likely to have greater improvements have clinical importance to determine 
patients who may have maximal benefits by astaxanthin. A randomized, controlled, and double-blinded study including an adequately powered sample size is required to test further hypothesis that there is a causal relationship between astaxanthin supplementation and improvements in the self-reported physical activity and HRQoL.

In conclusion, this sub-study of our previous observational pilot study found that following 3-month astaxanthin supplementation, improvements of the selfreported physical activity level were observed along with the improvements of HRQoL in both mental and physical components. Patients with higher baseline HR and poorer baseline physical activity and HRQoL in the mental component have potentials to have greater improvement of physical activity. Furthermore, patients with ischemic etiology of HF may have greater improvement of HRQoL in the physical component.

\section{Acknowledgments}

Funding: This study was supported by AstaReal Co., Ltd., and partly by a Grant-in-Aid for Scientific Research (Grant Number, 26507010); JSPS KAKENHI (Grant Number, JP17K09527); JSPS KAKENHI (Grant Number, JP18K15904); a grant to The Intractable Respiratory Diseases and Pulmonary Hypertension Research Group from the Ministry of Health, Labor and Welfare (H29-027); and MEXT*-Supported Program for the Strategic Research Foundation at Private Universities, 2014-2018 (*Ministry of Education, Culture, Sports, Science and Technology). These sources have no other roles in this study.

\section{Footnote}

Reporting Checklist: The authors have completed the TREND reporting checklist. Available at http://dx.doi. org/10.21037/apm-20-1378

Data Sharing Statement: Available at http://dx.doi. org/10.21037/apm-20-1378

Peer Review File: Available at http://dx.doi.org/10.21037/ apm-20-1378

Conflicts of Interest: All authors have completed the ICMJE uniform disclosure form (available at http://dx.doi. org/10.21037/apm-20-1378). Drs. Ishiwata, Sato, Kasai, and Naito are affiliated with a department endowed by
Philips, Fukuda Denshi, and ResMed. Dr. Daida received manuscript fees and research and scholarship funds from Kirin Co., Ltd.; Kaken Pharmaceutical Co., Ltd.; Abbott Japan Co., Ltd.; Astellas Pharma Inc.; AstraZeneca K.K.; Bayer Yakuhin Ltd.; Boston Scientific Japan K.K.; BristolMyers Squibb; Daiichi Sankyo Company; MSD K.K.; Pfizer Inc.; Philips; Sanofi K.K.; and Takeda Pharmaceutical Co., Ltd, AstaReal Co., Ltd. The other authors have no conflicts of interest to declare.

Ethical Statement: The authors are accountable for all aspects of the work in ensuring that questions related to the accuracy or integrity of any part of the work are appropriately investigated and resolved. This is a substudy of the prospective observational pilot study (UMINID: 000014088), which was approved by the Juntendo University Hospital Institutional Review Board (\#14019), and the study complied with the ethical principles of the Declaration of Helsinki (as revised in 2013). Written informed consent was obtained from all participants.

Open Access Statement: This is an Open Access article distributed in accordance with the Creative Commons Attribution-NonCommercial-NoDerivs 4.0 International License (CC BY-NC-ND 4.0), which permits the noncommercial replication and distribution of the article with the strict proviso that no changes or edits are made and the original work is properly cited (including links to both the formal publication through the relevant DOI and the license). See: https://creativecommons.org/licenses/by-nc-nd/4.0/.

\section{References}

1. Hussein G, Sankawa U, Goto H, et al. Astaxanthin, a carotenoid with potential in human health and nutrition. $\mathrm{J}$ Nat Prod 2006;69:443-9.

2. Okada Y, Ishikura M, Maoka T. Bioavailability of astaxanthin in Haematococcus algal extract: the effects of timing of diet and smoking habits. Biosci Biotechnol Biochem 2009;73:1928-32.

3. Park JS, Chyun JH, Kim YK, et al. Astaxanthin decreased oxidative stress and inflammation and enhanced immune response in humans. Nutr Metab (Lond) 2010;7:18.

4. Choi HD, Kim JH, Chang MJ, et al. Effects of astaxanthin on oxidative stress in overweight and obese adults. Phytother Res 2011;25:1813-8.

5. Aoi W, Naito Y, Sakuma K, et al. Astaxanthin limits exercise-induced skeletal and cardiac muscle damage in 
mice. Antioxid Redox Signal 2003;5:139-44.

6. Nakao R, Nelson OL, Park JS, et al. Effect of astaxanthin supplementation on inflammation and cardiac function in BALB/c mice. Anticancer Res 2010;30:2721-5.

7. Earnest CP, Lupo M, White KM, et al. Effect of astaxanthin on cycling time trial performance. Int J Sports Med 2011;32:882-8.

8. Tsutsui T, Tsutamoto T, Wada A, et al. Plasma oxidized low-density lipoprotein as a prognostic predictor in patients with chronic congestive heart failure. J Am Coll Cardiol 2002;39:957-62.

9. Kato T, Kasai T, Sato A, et al. Effects of 3-month astaxanthin supplementation on cardiac function in heart failure patients with left ventricular systolic dysfunction -a pilot study. Nutrients 2020;12:1896.

10. Hobbs FD, Kenkre JE, Roalfe AK, et al. Impact of heart failure and left ventricular systolic dysfunction on quality of life: a cross-sectional study comparing common chronic cardiac and medical disorders and a representative adult population. Eur Heart J 2002;23:1867-76.

11. Matsuo S, Imai E, Horio M, et al. Revised equations for estimated GFR from serum creatinine in Japan. Am J Kidney Dis 2009;53:982-92.

12. Aebischer CP, Schierle J, Schuep W. Simultaneous determination of retinol, tocopherols, carotene, lycopene, and xanthophylls in plasma by means of reversed-phase high-performance liquid chromatography. Methods Enzymol 1999;299:348-62.

13. Sasayama S, Asanoi H, Ishizaka S, et al. Evaluation of functional capacity of patients with congestive heart failure. In: Yasuda H, Kawaguchi H, editors. New aspects in the treatment of failing heart. Tokyo: Springer-Verlag; 1992. p. 113-7.

14. Fukuhara S, Bito S, Green J, et al. Translation, adaptation, and validation of the SF-36 Health Survey for use in Japan. J Clin Epidemiol 1998;51:1037-44.

15. Tokuda Y, Okubo T, Ohde S, et al. Assessing items on the SF-8 Japanese version for health-related quality

Cite this article as: Ishiwata S, Kato T, Kasai T, Sato A, Yatsu S, Matsumoto H, Shitara J, Murata A, Shimizu M, Suda S, Matsue Y, Naito R, Hiki M, Daida H. Changes in self-reported physical activity and health-related quality of life following 3-month astaxanthin supplementation in patients with heart failure: results from a pilot study. Ann Palliat Med 2021;10(2):1396-1403. doi: 10.21037/apm-20-1378 of life: a psychometric analysis based on the nominal categories model of item response theory. Value Health 2009; 12:568-73.

16. Benes J, Kotrc M, Borlaug BA, et al. Resting heart rate and heart rate reserve in advanced heart failure have distinct pathophysiologic correlates and prognostic impact: a prospective pilot study. JACC Heart Fail 2013;1:259-66.

17. Verbrugge FH, Vrijsen J, Vercammen J, et al. Heart rate reduction and exercise performance in recent onset heart failure with reduced ejection fraction: arguments for betablocker hypo-response. Acta Cardiol 2015;70:565-72.

18. Abdel-Salam Z, Rayan M, Saleh A, et al. I(f) current inhibitor ivabradine in patients with idiopathic dilated cardiomyopathy: Impact on the exercise tolerance and quality of life. Cardiol J 2015;22:227-32.

19. Hopper I, Connell C, Briffa T, et al. Nutraceuticals in Patients With Heart Failure: A Systematic Review. J Card Fail 2020;26:166-79.

20. Cicero AFG, Colletti A, von Haehling S, et al. Nutraceutical support in heart failure: a position paper of the International Lipid Expert Panel (ILEP). Nutr Res Rev 2020;33:155-79.

21. Hofman-Bang C, Rehnqvist N, Swedberg K, et al. Coenzyme Q10 as an adjunctive in the treatment of chronic congestive heart failure. The Q10 Study Group. J Card Fail 1995;1:101-7.

22. Adarsh K, Kaur H, Mohan V. Coenzyme Q10 (CoQ10) in isolated diastolic heart failure in hypertrophic cardiomyopathy (HCM). Biofactors 2008;32:145-9.

23. Martin HD, Ruck C, Schmidt M, et al. Chemistry of carotenoid oxidation and free radical reactions. Pure Appl Chem 1999;71:34630514.

24. Iwamoto T, Hosoda K, Hirano R, et al. Inhibition of lowdensity lipoprotein oxidation by astaxanthin. J Atheroscler Thromb 2000;7:216-22.

25. Fratter A, Biagi D, Cicero AFG. Sublingual Delivery of Astaxanthin through a Novel Ascorbyl Palmitate-Based Nanoemulsion: Preliminary Data. Mar Drugs 2019;17:508. 